\title{
A comparative study between atropine and tropicamide as cycloplegic agents for a sample of Iraqi children
}

\author{
Bahir A.R.Mshimesh PhD \\ Dept. of Pharmacology and Toxicology, College of Pharmacy, Al-Mustansiriya University, Baghdad, Iraq
}

\begin{abstract}
\end{abstract}
Background A perfect cycloplegic medication which is viable, nontoxic, and helpful permitting precise estimation of the refractive errors by objective and/ or subjective tests isn't yet accessible

Objective This trial was designed to evaluate the cycloplegic activity and adverse effects of two cycloplegic agents (atropine vs. tropicamide) for children with hyperopia. The response to cycloplegia in different age groups, with or without strabismus, was also compared.

Methods Tropicamide $1 \%$ eye drops (Regimen 1 ) and Atropine 1\% eye drops (Regimen 2) was evaluated in thirty children with different ages. Cycloplegic refractions and adverse effects were assessed. The results expressed refractions and presented as mean \pm SD. A P-value of less than 0.05 was considered statistically significant.

Results Tropicamide refraction mean value $(+3.60 \pm 2.25 \mathrm{D})$ didn't differ significantly in comparison with that of atropine $(+3.92 \pm 2.50 \mathrm{D})$; $(P>0.05)$. Children during regimen 2 (atropine drops) suffered from more frequent and statistically significant side effects $(p<0.05)$, represented by blurred vision, fever; flushing and tachycardia, compared with regimen 1 (tropicamide drops).

Conclusion Tropicamide indicated to children with different ages is adequate to deliver great cycloplegia, with activity approximate to and less toxic than atropine, even in kids with a high level of hyperopia, with or without eye squint.

Keywords Cycloplegia; Hyperopia; Atropine; Tropicamide.

List of abbreviation: $\mathrm{D}=$ diopter, $\mathrm{RT}=$ right, $\mathrm{LT}=$ left, $\mathrm{SD}=$ standard deviation.

\section{Introduction}

A $s$ a definition, the visual accommodative intensity is the power of settlement that adjusts way of the light beams via making ciliary muscles modifying flow of the focal point ${ }^{(1)}$.

Muscarinic receptors of the smooth muscle innervated by parasympathetic nervous system are available in the ciliary body. Cycloplegia restrains accommodative intensity by hindering activity of the ciliary body, permitting the targeted refractive error to be estimated. This anticholinergic activity hinders cholinergic properties (muscarinic receptors) of the iris sphincter, leading to mydriasis, and ciliary muscle, leading to cycloplegia (2).

Assessment of cycloplegic effect not just enables refraction errors to be resolved; they additionally expand the eye pupil, setting up the eyes for perfect ophthalmoscopic evaluation. Cycloplegic refraction is essential for assessment of eyes with visual weakness and deviation. It additionally helps discovery of complete hyperopia that related to accommodative esotropia and counteracts the false results in nearsighted conditions ${ }^{(3)}$. 
After the disclosure of current laser refractive medical procedure, cycloplegic refraction has turned into a profitable preoperative measure for precisely deciding the refractive errors. In children with different ages, refraction under cycloplegia will affirm conclusion of accommodative fit, that is a consistent or discontinuous automatic increment in ciliary withdrawal. Patients with low hyperopia might be displayed as nearsighted during test; this pseudo nearsightedness (pseudomyopia) can be distinguish by cycloplegic assessment ${ }^{(4,5)}$.

Cycloplegia also utilized in pharmacological occlusion treatment where eye with nonamblyopia is adequately hypermetropic and the successful obscuring of vision can be acquired by receiving a cycloplegics in just this eye. If during examination the patient switched from utilizing the normal eye to lazy one, chance is great that he or she will likewise do as such under therapy and that punishment will drive the amblyopic eye to be utilized ${ }^{(6)}$.

Therapeutic uses for refraction under cycloplegia are constrained in adulthood. As the abundance of accommodated eye steadily diminishes with life stages, an approximate matching among cycloplegic and refraction discovery will happen ${ }^{(7)}$.

The 3 most usually utilized cycloplegics, atropine, cyclopentolate, and tropicamide work by contending with the muscarinic mediator "acetylcholine", bringing about hindrance of contraction within muscles of ciliary body ${ }^{(8)}$. Various investigations have reported the viability of these cycloplegic drugs. A few researchers have demonstrated a noteworthy and others demonstrated no huge cycloplegic impact for a portion of these medications ${ }^{(9,10)}$.

Most clinicians concur that cycloplegia is essential during achieving refractive examination in kids, significant hyperopia, and those suffering squint ${ }^{(11)}$. Fogging and different procedures can't trade cycloplegic technique for exactness in deciding refractive error in early adolescence since it doesn't rely upon patient collaboration or fixation distance ${ }^{(12,13)}$.
The unaccepted idea of receiving eye drops, particularly in children, can anticipate achieving eye examination and is important to the point that sprays have been recommended rather than eye drops by few researchers. Insufficient cycloplegia can make imprecise refraction and leads to improper demonstrative and remedial methodologies. On the contrary side, over dose of cycloplegics may cause adverse drug effects or prompt patient discomfortable status ${ }^{(14)}$.

Atropine, the most familial cycloplegic known utilized in children, has its own characteristic features and drawbacks, as long-term activity. A few specialists have demonstrated atropine as the first powerful agent in cancelling accommodative reflex in youthful esotropic kids. Much of the time of esotropia associated with hypermetropia in kids under 5 years old, atropine and scopolamine ideally is utilized to guarantee that no remaining accommodative effect still masked $(15,16)$.

Atropine is the best quality level for finishing cycloplegia, however, it needs no less than 3 hours to achieve maximum impact and should be utilized for 3 days to deliver the required cycloplegia. It takes 1 to 2 weeks for its impact to clear from the ciliary bodies and pupil. Tropicamide, again, has a quicker beginning of activity and achieves maximum impact following 30-45 minutes; its cycloplegic impact clears following 6-8 hours and has less adverse reactions (17). All visual medications have unfortunate symptoms, separating into those of systemic and visual in nature. Be that as it may, the undesirable effects from cycloplegic and mydriatic agents are uncommon, contrasted with their broad uses ${ }^{(18)}$.

The poisonous impacts of atropine might be outlined by saying: "visually impaired like a bat; extremely dry like a bone; mad like a hatter; and red like a beetroot ". Atropine utilizers are "visually impaired" inferable from the cycloplegic effects; "dry" because of the hindrance of the perspiration and salivary organs; "mad" attributable to the consequences for the CNS; and "red" on account of vasodilatation and the absence of 
capacity for the perspiration organs (19). Atropine may prompt undesirable effects, for example, hyperthermia, tachycardia, seizures, and even loss of life ${ }^{(20)}$.

The perfect cycloplegic medication that is potent, non-toxic and permitting exact estimation of dioptric error via subjective or objective methods isn't yet accessible. This study was designed to compare the cycloplegic activity and adverse effects between $1 \%$ eye drops of atropine versus $1 \%$ eye drops of tropicamide for a sample of Iraqi children with hyperopia.

\section{Methods}

This study involved 30 children (16 males/ 14 females) with range (2-9 years) and hyperopia $>1.0$ Diopter (unit of refraction power) in one eye as minimum inclusion criteria, registered in a prospective trial for kids visiting Ibn ALHaitham Teaching Hospital and private eye clinics, during January to April of 2014 under supervision of professional ophthalmologists and approval of ethical committee in the College of Pharmacy / Mustansiryah University, after taking an oral consent from the parents of the participated children.

The primary goal of this study involved a comparison between tropicamide and atropine in cycloplegic effect, onset and duration of action, and their safety. The influence of age, sex, and iris color on the completeness of cycloplegia was considered as a secondary goal.

Because of the expected atropine pharmacokinetic profiles and long half-life, its onset and duration of action were detected by an accurate follow up for mydriatic and cycloplegic effects with the traditional slit lamp by giving a restricted appointment of the enrolled children for ophthalmic investigation depending on the doctor instructions.

The selection of the sample was based on the inclusion criteria which involved children below ten years, with approximate male/female ratio, suffering from hypermetropia with or without strabismus, and having different iris colors. Kids prohibited from this examination include those with known cardiovascular disorders, ophthalmic ailment other than refractive diseases, as well as squint, history of hypersensitivity to the studied agents, and those with non compliance to the treatment protocol. All participants experienced a routine visual assessment.

Each child was given two regimens of eye drops, regimen 1 (tropicamide as mydriacyl ${ }^{\circledR} /$ Alcon Lab.), and then after one week, regimen 2 (atropine as isopto ${ }^{\circledR}$ atropine/ Alcon Lab.). Tropicamide $1 \%$ ophthalmic drops given at the hospital or eye centers in the conjunctival cyst twice at interims of 5 min upon testing time; refraction tested $0.5 \mathrm{hr}$ after the last dropping by advanced retinoscopy. Atropine $1 \%$ ophthalmic drop given at the home 2 times/day for 3 days before and upon the time of test. After instillation, lacrimal puncti was shut via compress above medial canthus during about $3 \mathrm{~min}$ to limit distribution. Guardians educated for findings of ocular and peripheral reactions because of medications and were told to restore kids in the center if undesired occasions were reported.

The results of refractive error were expressed as mean \pm SD. Student t-test and chi- square test were used for statistical analysis, applying the Microsoft Office Excel Program-2007. A Pvalue of less than 0.05 was considered statistically significant.

\section{Results}

A total of 30 children (60 eyes) with age mean of (5.47 \pm 1.44 years), range (2-9 years) were prospectively involved. Refractions were read post cycloplegic effects with regimen 1 (tropicamide 1\%) and regimen 2 (atropine 1\%) (table 1). The mean of tropicamide refraction values $(+3.60 \pm 2.25 \mathrm{D})$ didn't differ significantly in comparison with that of atropine $(+3.92 \pm$ $2.50 \mathrm{D})$; ( $\mathrm{P}>0.05$ ) (table 2$)$. 
Mshimesh, A Comparative Study between Atropine and Tropicamide ...

Table (1): Cycloplegic refractions and their difference values.

\begin{tabular}{|c|c|c|c|c|}
\hline $\begin{array}{l}\text { Pt. } \\
\text { No. }\end{array}$ & $\begin{array}{c}\text { Tropicamide drops } \\
\text { Rt/Lt eye }\end{array}$ & $\begin{array}{l}\text { Atropine drops } \\
\text { Rt/Lt eye }\end{array}$ & $\begin{array}{l}\text { Difference } \\
\text { Values }\end{array}$ & $\begin{array}{c}\text { Age } \\
\text { (years) }\end{array}$ \\
\hline 1 & $+2.5 /+2.5$ & $+2.5 /+2.5$ & $0.0 / 0.0$ & 2 \\
\hline 2 & $+4.75 /+4.75$ & $+5.25 /+5.25$ & $0.5 / 0.5$ & 4.5 \\
\hline 3 & $+6.0 /+6.0$ & $+7.0 /+7.0$ & 1.0/1.0 & 3 \\
\hline 4 & $+3.25 /+3.25$ & $+4.0 /+4.0$ & $0.75 / 0.75$ & 7 \\
\hline 5 & $+8.0 /+8.5$ & $+8.5 /+8.5$ & $0.5 / 0.0$ & 5 \\
\hline 6 & $+1.5 /+1.5$ & $+1.5 /+1.5$ & $0.0 / 0.0$ & 9 \\
\hline 7 & $+4.0 /+4.0$ & $+4.0 /+4.0$ & 0.0/0.0 & 6 \\
\hline 8 & $+2.75 /+2.75$ & $+3.0 /+3.0$ & $0.25 / 0.25$ & 5.5 \\
\hline 9 & $+2.5 /+2.5$ & $+2.5 /+2.5$ & $0.0 / 0.0$ & 5 \\
\hline 10 & $+4.25 /+5.0$ & $+4.50 /+5.50$ & $0.25 / 0.50$ & 9 \\
\hline 11 & $+1.0 /+1.0$ & $+1.0 /+1.0$ & $0.0 / 0.0$ & 5 \\
\hline 12 & $+3.0 /+3.0$ & $+3.5 /+3.5$ & $0.5 / 0.5$ & 5 \\
\hline 13 & $+2.75 /+2.50$ & $+3.0 /+3.0$ & $0.25 / 0.50$ & 8 \\
\hline 14 & $+4.0 /+4.5$ & $+4.5 /+5.0$ & $0.5 / 0.5$ & 6 \\
\hline 15 & $+4.5 /+4.5$ & $+5.0 /+5.0$ & $0.5 / 0.5$ & 6 \\
\hline 16 & $+3.0 /+3.0$ & $+3.25 /+3.25$ & $0.25 / 0.25$ & 5 \\
\hline 17 & $+3.0 /+3.25$ & $+3.0 /+3.50$ & $0.0 / 0.25$ & 7.5 \\
\hline 18 & $+3.25 /+3.25$ & $+3.25 /+3.25$ & $0.0 / 0.0$ & 5 \\
\hline 19 & $+3.75 /+3.75$ & $+4.0 /+4.0$ & $0.25 / 0.25$ & 8 \\
\hline 20 & $+3.0 /+3.0$ & $+3.5 /+3.5$ & $0.5 / 0.5$ & 2.5 \\
\hline 21 & $+1.0 /+2.0$ & $+1.0 /+2.0$ & $0.0 / 0.0$ & 5.5 \\
\hline 22 & $+7.0 /+7.0$ & $+8.0 /+8.0$ & $1.0 / 1.0$ & 7 \\
\hline 23 & +3.5/+3.5 & $+4.0 /+4.0$ & $0.5 / 0.5$ & 3 \\
\hline 24 & $+3.25 /+3.25$ & $+3.5 /+3.5$ & $0.25 / 0.25$ & 4 \\
\hline 25 & $+3.75 /+4.25$ & $+4.0 /+4.5$ & $0.25 / 0.25$ & 8.5 \\
\hline 26 & $+5.0 /+5.0$ & $+6.0 /+6.0$ & 1.0/1.0 & 4 \\
\hline 27 & $+3.5 /+3.5$ & $+4.0 /+4.0$ & $0.5 / 0.5$ & 6.5 \\
\hline 28 & $+4.5 /+4.5$ & $+5.0 /+5.0$ & $0.5 / 0.5$ & 4 \\
\hline 29 & $+2.5 /+2.5$ & $+2.5 /+2.5$ & $0.0 / 0.0$ & 3.5 \\
\hline 30 & $+6.0 /+6.0$ & $+6.0 /+6.0$ & $0.0 / 0.0$ & 9 \\
\hline
\end{tabular}

Data represented by Diopters (D)

Children were distributed depending to their ages into 2 groups, either $\leq$ or $>6$ years. In younger ages, the cycloplegic refraction mean post atropine instillation was $(+4.15 \pm 2.88 \mathrm{D})$ and for tropicamide was $(+3.77 \pm 2.68 \mathrm{D})$; $(\mathrm{P}$ $>0.05)$. In older ages, the cycloplegic refraction mean post atropine instillation was $(+3.70 \pm$ $1.87 \mathrm{D})$ and for tropicamide was $(+3.37 \pm 1.56$ D); $(P>0.05)$.

In hypermetropic-strabismus kids, the mean cycloplegic refraction post atropine drops was
$(+4.0 \pm 2.25 \mathrm{D})$ and by tropicamide was $(+3.70$ $\pm 2.87 \mathrm{D})$; ( $P>0.05)$. In hypermetropic kids without strabismus, the mean cycloplegic refraction post atropine drops was $(+3.82 \pm$ $1.97 \mathrm{D})$ and by tropicamide was $(+3.45 \pm 1.87$ D); $(P>0.05)$.

Regarding gender, no significant association was found between the sex and refractive error values. The role of iris color on accommodative eye after receiving cycloplegic agent was investigated via distributing 5 known iris color 
classes into two categories: a light-iris and a dark-iris. Class 1, 2, 3 were joined as the lightiris group and comprised of irises that were blue; gray; green; or light brown, with or without brown or yellow pigmentation. Class 4 and 5 were brown or dark brown with negligible yellow pigmentation and were named the dark-iris group. In this study, no association was seen between residual accommodation and iris color.

Table (2): means of refractive error for each subgroup

\begin{tabular}{|c|c|c|c|c|}
\hline Stratification & No. & Tropicamide drops & Atropine drops & p-value \\
\hline All participants & 30 & $+3.60 \pm 2.25$ & $+3.92 \pm 2.50$ & $P=0.071$ \\
\hline Children $\leq 6$ years & 20 & $+3.77 \pm 2.68$ & $+4.15 \pm 2.88$ & $P=0.096$ \\
\hline Children $>6$ years & 10 & $+3.37 \pm 1.56$ & $+3.70 \pm 1.87$ & $P=0.085$ \\
\hline $\begin{array}{l}\text { Hypermetropic } \\
\text { with strabismus }\end{array}$ & 18 & $+3.70 \pm 2.87$ & $+4.0 \pm 2.25$ & $P=0.073$ \\
\hline $\begin{array}{c}\text { Hypermetropic } \\
\text { without strabismus }\end{array}$ & 12 & $+3.45 \pm 1.87$ & $+3.82 \pm 1.97$ & $P=0.094$ \\
\hline
\end{tabular}

The onset of mydriatic and cycloplegic effects for tropicamide was faster than that of atropine, while the duration of mydriatic and cycloplegic effects for atropine was longer than for tropicamide (table 3).

Adverse effects of these cycloplegic agents were summarized in table (4). Children using regimen 2 (atropine drops) suffered from more frequent and statistically significant side effects $(p<0.05)$, represented by blurred vision, fever; flushing and tachycardia, compared with regimen 1 (tropicamide drops).

Table (3): Onset and duration of action for the studied cycloplegic drugs

\begin{tabular}{clccc}
\hline & \multicolumn{2}{c}{ Onset of action } & \multicolumn{2}{c}{ Duration of action } \\
Drug & Mydriasis & Cycloplegia & Mydriasis & Cycloplegia \\
\hline Tropicamide & $15-30 \mathrm{~min}$ & $25-30 \mathrm{~min}$ & $4-6 \mathrm{hr}$ & $5-6 \mathrm{hr}$ \\
Atropine & $30-40 \mathrm{~min}$ & $1-1.5$ day & $7-10$ day & $12-14$ day \\
\hline
\end{tabular}

\section{Discussion}

The perfect cycloplegic substance should create full cycloplegia with insignificant adverse reactions and permit quick accommodation recovery. For kids who are at the period of visual development and have extensive eye accommodation going about as block against precise refraction, complete cycloplegia is a fundamental strategy in the finding and management of those patients ${ }^{(21)}$.
After atropine instillation, long-term cycloplegic effect at certain period may, sometimes, potentiate the probability of amblyopia. Besides, the guardians frequently experience obstacles in giving the drops in a perfect way and questions regularly emerge whether a maximum dilatation impact was accomplished ${ }^{(22)}$. 
Mshimesh, A Comparative Study between Atropine and Tropicamide ...

Table (4): Adverse effects for the studied cycloplegic drugs.

\begin{tabular}{cccc}
\hline Adverse effects & Tropicamide drops (1\%) & Atropine drops (1\%) & p-value \\
\hline Stinging & $2(6.6)$ & $3(10)$ & $\mathrm{P}=0.087$ \\
Blurred vision & $1(3.3)$ & $4(13.3)$ & $\mathrm{P}=0.046$ \\
photophobia & $3(10)$ & $2(6.6)$ & $\mathrm{P}=0.079$ \\
Fever & $0(0.0)$ & $3(10)$ & $\mathrm{P}=0.041$ \\
Dryness of skin & $0(0.0)$ & $1(3.3)$ & $\mathrm{P}=0.092$ \\
Flushing & $1(3.3)$ & $5(16.7)$ & $\mathrm{P}=0.026$ \\
Headache & $2(6.6)$ & $2(6.6)$ & $\mathrm{P}=0.065$ \\
Tachycardia & $0(0.0)$ & $3(10)$ & $\mathrm{P}=0.037$ \\
\hline
\end{tabular}

Data represented by numbers (\%).

P-value $<0.05$ considered statistically significant.

Numerous ophthalmologists trust that other cycloplegics, as tropicamide; cyclopentolate; and homatropine alone are insufficient in kids with 2-5 years of age, particularly in esotropic kids with hyperopia $>2.0$ diopters, who usually should receive atropine drops for refraction and to recognize the covered hyperopia (23). Others have exhibited that the cycloplegic impact of these medications is similar to atropine ${ }^{(24)}$.

Such huge number of researchers regarded atropine substance as the medication of decision for full cycloplegia and trust that other mydriatic substances can't be suitable options. Notwithstanding, because of its disadvantages, the troublesome course, and delayed disability of near vision, atropine continuously may switch by other cycloplegic drugs which have less adverse events, less demanding to monitor, and have shorter term of activity (25). Comparisons of mixing these medications have likewise unsuccessful to determine the perfect regimen. Possible foundations for the different outcomes could be the variation in medications to be combined, treatment regimens, and individual variations (26).

In the present study, the mean of tropicamide refraction values $(+3.60 \pm 2.25 \mathrm{D})$ didn't differ significantly in comparison with that of atropine $(+3.92 \pm 2.50 \mathrm{D}) ;(P>0.05)$. Both cycloplegics in the current trial were notably matched; even in kids with extensive hypermetropia. So, it may possible to shift home atropinization to tropicamide administration in the eye clinics or specialized hospitals.

For medications like tropicamide and cyclopentolate, caution ought to be taken as they will in general be less cycloplegic than atropine in youthful kids with sever hypermetropia. In any case, atropine ought to be held for kids (under 6 years) with extensive accommodative esotropia and those with previous tropicamide sensitivity (27). This trial reported that $1 \%$ eye drops of tropicamide, 5 minutes separated, give impressive cycloplegia adequate to refraction in almost all children.

Certain research was accomplished on esotropic kids less than 5 years with atropine and other cycloplegic drugs, and demonstrated that atropine was likely pointless (28). Interestingly, another examination reported atropine to revealed (0.35-0.45) diopter higher hyperopia in kids more youthful than 6 years of age ${ }^{(27)}$. For cycloplegic refraction, an allowance must be achieved for cancelled ciliary body tone; this estimation is considered as 1.0 diopter on account of atropine. Few authors suggested an equivalent tonus allowance for atropine drops and other cycloplegic medications; while other researchers recommended that an allowance for other cycloplegics, except for atropine, is improper (29). In kids with a high level of hypermetropia, cycloplegics other than atropine didn't create a total cycloplegia, where $22 \%$ of the kids had an 
extra hyperopia of $(+1.0)$ diopter or more which was discovered by atropine ${ }^{(30)}$.

In this study and for younger children, the cycloplegic refraction mean after atropine $(+4.15 \pm 2.88 \mathrm{D})$ didn't differ statistically from that of tropicamide $(+3.77 \pm 2.68 \mathrm{D})$; $(\mathrm{P}>0.05)$. Considering older children, the cycloplegic refraction mean after atropine $(+3.70 \pm 1.87 \mathrm{D})$ also didn't differ statistically from that of tropicamide $(+3.37 \pm 1.56 \mathrm{D}) ;(P>0.05)$. Just four eyes, every one belong to $<6$ years old kids, which had 1.0 diopter hyperopia discovered by atropine, not tropicamide. The significance of cycloplegic atropinization in assessment of strabismus kids was referenced in previous studies ${ }^{(4)}$. The current investigation demonstrated that, for kids with or without squint, refractive cycloplegia post atropine and tropicamide was approach $(+4.0 \pm 2.25 \mathrm{D}$ and $+3.82 \pm 1.97 \mathrm{D}$ vs. $+3.70 \pm 2.87 \mathrm{D}$ and $+3.45 \pm$ $1.87 \mathrm{D}$, respectively). In this way, tropicamide may regard as the decision of cycloplegia for kids with or without squint.

In spite of the fact that researchers have detailed a relationship between iris coloration and residual accommodation, which is identified with the ethnicity, no such relation was observed in this examination. A marked correlation between ethnic factor and residual accommodation was reported in the past examinations, where a critical contrast in remaining accommodation between the Hispanic and white populaces was accounted for ${ }^{(25)}$.

In spite of the fact that atropine provides adequate cycloplegia, the adverse effects and persistent duration of action have encouraged a search for alternate cycloplegic agents. Caution must be taken about the incidence of transient psychosis which may observe even after instillation of atropine drops. Other neurological toxicities, including seizure and delirium, can also occur with atropine ${ }^{(31)}$. The probability of adverse events for investigational ocular agents was reported to be less than $1 \%$, where the authors studied a huge number of dilating drops, ranged from weak to powerful agents. Serious adverse drug reactions have additionally reported, like tremor, increasing heart rate, and confusion of the CNS ${ }^{(32)}$.

In the present study, children who received tropicamide (regimen 1) didn't show significant side effects when compared with that of atropine (regimen 2), where $13.3 \%, 10 \%, 16.7$ $\%$, and $10 \%$ of children during atropine drops suffering from blurred vision, fever, flushing, and tachycardia, respectively. The small sample size used may affect on the incidence of these anticholinergic problems.

To achieve complete cycloplegia and to avoid the complications and morbidity of atropine, different formulas with different components and concentrations of these medications have been prepared. The major explanation behind utilizing a diminished concentration of atropine in more youthful kids was to decrease the frequency of atropine adverse reactions in those patients. This seemed confounding in light of the fact that more youthful kids have a more prominent accommodative reaction (33). From the pharmacokinetic point of view, and as an advantage over atropine, tropicamide instillation has a rapid onset, short duration of action, and can be given at the time of examination in the eye clinic or hospital. The greatest cycloplegic impact is achieved following 30 minutes and stays stable for over 120 minutes (34). As burdens with home atropinization, compliance is frequently not satisfied, the medication impacts are longacting and usually hard to choose whether a total cycloplegic impact has been accomplished (35). Results of the current study were consistent with this fact, where tropicamide was faster in onset, while atropine was longer in its duration of action.

From above, and because of its disadvantages, troublesome regimen, and delayed weakness of near vision, atropine ought to be switched by other cycloplegic medications which have less adverse reactions, less demanding to monitor, and has a shorter term of activity. 
Taking everything into account, one can conclude that tropicamide used for children with different ages is adequate to create great cycloplegia, with an impact close to and less toxic than atropine, even in kids with a high level of hypermetropia, with or without squint. Likewise, the residual eye accommodation was not related with life stage, sex, or color of iris, recommending that these elements might be of no worry when utilizing tropicamide or atropine in those patients.

\section{Acknowledgement}

The author would like to thanks the doctors and staff of Ibn AL-Haitham Teaching Hospital for their help and support to achieve this study. Special thanks also introduce to the $5^{\text {th }}$ stage students, Ruqaia and Hawraa, in the College of Pharmacy, Al-Mustansiriyah University.

\section{Conflict of interest}

The author declares no conflict of interest.

\section{Funding}

None.

\section{References}

1. Aneja, A. Ophthalmic Drugs; Diagnostic and Therapeutic. In G. Hopkins \& R. Pearson (eds). Ophthalmic Drugs: Diagnostic and Therapeutic Uses. Philadelphia, PA: Elsevier, 2007; pp 506-510.

2. Mindel JS. Cholinergic pharmacology In: Tasman W, Jaeger EA, eds. Duane's Foundations of Clinical Ophthalmology, Philadelphia: JB Lippincott,Vol. 3, 2010, p345.

3. Zetterstrom C, Hahnenberger R. Pharmacological characterization of human ciliary muscle adrenoceptors in vitro. Exp Eye Res, 1988, 46: 421430.

4. Caloroso EE, Rouse MW, Cotter SA. Clinical Management of Strabismus. Boston: ButterworthHeinemann, 2012, pp 14-17.

5. Lograno MD, Reibaldi $A$. Receptor-responses in fresh human ciliary muscle. Br J Pharmacol, 1986, 87: 379385.

6. Peyman GA, Saunders DR, Goldberg MF. Principles and Practice of Ophthalmology. Philadelphia: WB Saunders, 2014; 201-204.

7. Rengstorff RH, Doughty CB. Mydriatic and cycloplegic drugs: A review of ocular and systemic complications. Am J Optom Physiol Opt, 1982, 59: 162-177.
8. Banks, M. S. The Development of Visual Accommodation during Early Infancy. Child Development, 1980; 51, 646-666.

9. Bujara K, Schulz E, Haase W. Retinoscopy under cycloplegic and non-cycloplegic conditions in children comparison of measurements of three examiners. Albrecht Von Graefes Arch Klin Exp Ophthalmol, 1981, 216: 339-343.

10. Duke- Elder, Duke-Elder SS. Duke Elder's Practice of Refraction. 12th ed., B. I. Churchill Livingstone, New Delhi, 2006, p71.

11. Kleinstein RN, Mutti DO, Manny RE, Shin JA, Zadnik K. Cycloplegia in African-American children. Optom Vis Sci, 1999, 76: 102-107.

12. Chan, O. Y. C., \& Edwards, M. Comparison of cycloplegic and noncycloplegic retinoscopy in Chinese preschool children. Journal of American Academy of Optometry, 1993; 71(5), 312-318.

13. Vitale A, Foster CS. Mydriatic and cycloplegic agents. In: Zimmerman TJ, Kooner K, Sharir M, Fechtner R. Text book of Ocular Pharmacology. Philadelphia: Lippincott Williams \& Wilkins, 2013, pp 703-711.

14. Wong CY, Fan DS, Yu CB, Lam DS. Topical mydriatic and cycloplegic spray for Chinese children. J Pediatr Ophthalmol Strabismus, 2003, 40: 349-352.

15. Havener WH. Ocular Pharmacology. 11th ed. The C. V. Mosby Company, Saint Louis, 2011, p 253.

16. Sheie, H. G., \& Albert, D. M. Adless Textbook of Ophthalmology. 15th Edition. Philadelphia: W. B. Saunders, 2009, p45.

17. Newell, F. W. Ophthalmology: Principle and Concepts. 15th Edition, St. Louis, Toronto: Princetown, 2012, p86.

18. Applebaum $M$, Jaanus SD. Use of diagnostic pharmaceutical agents and incidence of adverse effects. Am J Optom Physiol Opt, 1983, 60: 384-388.

19. Siderov J, Nurse $S$. The mydriatic effect of multiple doses of tropicamide. Optom Vis Sci 2005;82:955-8.

20. Rengstoff, R. H., \& Doughty, C. B. Mydriatic and Cycloplegic Drugs: A Review of Ocular and Systemic Complications. Am. J. Optom. Physio. Opt., 1982; 59(2).

21. Rseenfield, M., \& Linfield, P. B. A Comparison of the Effects of Cycloplegics on Accommodation Ability for Distant Vision and Apparent Near Point. Ophthal. Physio. Opt., 1986; 6, 317 - 320.

22. Rosenbaum AL, Bateman JB, Bremer DL, Liu PY. Cycloplegic refraction in esotropic children. Cyclopentolate versus atropine. Ophthalmology, 1981, 88: 1031-1034.

23. Lin LL, Shih YF, Hsiao CH, Su TC, Chen CJ, et al. The cycloplegic effects of cyclopentolate and tropicamide on myopic children. J Ocul Pharmacol Ther, 1998, 14: 331-335.

24. Portney, G. L., \& Purcell T. W. The Influence of Tropicamide in Intraocular Pressure. Am. J. Ophthalmol, 1995; 7, 31.

25. Richardson, L. C. D. R. Comparing the Mydriatic Effects of Tropicamide with Respect to Iris Pigmentation. J. AOA., 1982; 53(4). 
26. Krumholz DM, Portello JK, Rosenfield M, Rosenbaum JD. A combination solution for routine pupillary dilation. Optometry 2006;77:350-3.

27. Goldstein JH, Schneekloth BB. Atropine versus cyclopentolate in esodeviations. Ophthalmic Surg Lasers, 1996, 27: 1030-1034.

28. Celebi S, Aykan U. The comparison of cyclopentolate and atropine in patients with refractive accommodative esotropia by means of retinoscopy, auto refractometry, and biometric lens thickness. Acta Ophthalmol Scand, 1999, 77: 426-429.

29. Blansett DK. Dilation of the pupil. In: Bartlett JD, Jannus SD. Clinical Ocular Pharmacology, 4th ed. Boston: Butterworth, 2001:405-11.

30. Kawamoto K, Hayasaka S. Cycloplegic refractions in Japanese children: a comparison of atropine and cyclopentolate. Ophthalmologica, 1997, 211: 57-60.
31. Twa MD, Bailey MD, Hayes J, Bullimore M. Estimation of pupil size by a digital photograph. J Cataract Refract Surg 2004; 30:381-9.

32. Pop M, Payette $Y$, Santoriello E. Comparison of the pupil card and pupillometer in measuring pupil size. J Cataract Refract Surg 2002;28:283-8.

33. Levine, L. Mydriatic Effectiveness of Dilue Combinations of Phenylephrine and Tropicamide. Am. J. Optom. And Physio. Opt., 1982; 59(7), 580594.

34. Manny, R. E., Hussein, M., Scheiman, M., Kurtz, D., Niemann, K., Zinzer, K., \& et al. Tropicamide (1\%): An Effective Cycloplegic Agent for Myopic Children. Investigative Ophthalmology \& Visual Science, 2001; 42(8), 1728-1735.

35. Davies, P. H.The actions and uses of ophthalmic drugs. 14th Ed. Butterworths, England, 2005; 80, pp 96-104.

E-mail: dr.bahirrazzaq@gmail.com www.uoMustansiriyah.edu.iq Received 22 ${ }^{\text {nd }}$ Dec. 2015: Accepted $12^{\text {th }}$ June 2016 